\title{
The awareness of the scared - context dependent influence of oxytocin on brain function
}

\author{
Baettig, Linda ; Baeumelt, Andreas ; Ernst, Jutta ; Boeker, Heinz ; Grimm, Simone ; Richter, Andre
}

\begin{abstract}
Oxytocin is both a hormone and a neurotransmitter and has been originally recognized for its role in childbirth and lactation. Later, it became widely known as a "cuddle hormone" that induces trusting behavior towards strangers and reduces social stress and anxiety. Several studies showed that oxytocin influences empathic behavior and has prosocial effects. The anterior cingulate cortex and the anterior insula are brain regions that are active when humans observe fear in others. Therefore, the aim of this study was to investigate whether oxytocin administration affects activity in these regions depending on whether a threat is directed at another person (empathy) compared to when the threat is directed at the subject itself (fear). Our findings demonstrate increased anterior cingulate cortex activation after oxytocin administration in the fear, but not in the empathy condition. Furthermore, oxytocin administration was associated with deceased anterior insula activity in the empathy condition. However, our findings do not support the idea that oxytocin generally augments activity in brain regions associated with empathy. Thereby this study supports current research questioning that oxytocin has exclusively prosocial effects on human behavior. Rather, the effect of oxytocin depends on various contextual (e.g. presence of a familiar person) and interindividual (e.g. sex, mental disorder) factors. Therefore, to consider oxytocin an empathy inducing hormone is an oversimplification and future research should focus on factors moderating oxytocin effects.
\end{abstract}

DOI: https://doi.org/10.1007/s11682-019-00143-2

Posted at the Zurich Open Repository and Archive, University of Zurich

ZORA URL: https://doi.org/10.5167/uzh-185351

Journal Article

Accepted Version

Originally published at:

Baettig, Linda; Baeumelt, Andreas; Ernst, Jutta; Boeker, Heinz; Grimm, Simone; Richter, Andre (2020). The awareness of the scared - context dependent influence of oxytocin on brain function. Brain imaging and behavior, 14(6):2073-2083.

DOI: https://doi.org/10.1007/s11682-019-00143-2 


\section{The awareness of the scared - context dependent influence of oxytocin on brain function}

Bättig, Linda1 ${ }^{1}$ Baeumelt, Andreas², Ernst, Jutta², Boeker, Heinz², Grimm, Simone $2,3,4, *$ Richter, Andre ${ }^{1, *}$

${ }^{1}$ Department of Consultation-Liaison-Psychiatry and Psychosomatic Medicine, University Hospital Zurich, University of Zurich, Switzerland

2 Department of Psychiatry, Psychotherapy, and Psychosomatics, University Hospital of Psychiatry Zurich, Switzerland

${ }^{3}$ Department of Psychiatry, Charité, Campus Benjamin Franklin, Berlin, Germany

${ }^{4}$ Medical School Berlin, Berlin, Germany

* These authors contributed equally

Corresponding Author:

Andre Richter, M.D.

Department of Consultation-Liaison-Psychiatry and Psychosomatic Medicine University Hospital Zurich

Raemistrasse 100, 8091 Zurich, Switzerland

Telephone: +41442551639

andre.richter@uzh.ch 


\section{Abstract}

Oxytocin is both a hormone and a neurotransmitter and has been originally recognized for its role in childbirth and lactation. Later, it became widely known as a "cuddle hormone" that induces trusting behavior towards strangers and reduces social stress and anxiety. Several studies showed that oxytocin influences empathic behavior and has prosocial effects. The anterior cingulate cortex and the anterior insula are brain regions that are active when humans observe fear in others. Therefore, the aim of this study was to investigate whether oxytocin administration affects activity in these regions depending on whether a threat is directed at another person (empathy) compared to when the threat is directed at the subject itself (fear). Our findings demonstrate increased anterior cingulate cortex activation after oxytocin administration in the fear, but not in the empathy condition. Furthermore, oxytocin administration was associated with deceased anterior insula activity in the empathy condition. However, our findings do not support the idea that oxytocin generally augments activity in brain regions associated with empathy. Thereby this study supports current research questioning that oxytocin has exclusively prosocial effects on human behavior. Rather, the effect of oxytocin depends on various contextual (e.g. presence of a familiar person) and interindividual (e.g. sex, mental disorder) factors. Therefore, to consider oxytocin an empathy inducing hormone is an oversimplification and future research should focus on factors moderating oxytocin effects.

\section{Key Words}

empathy; oxytocin; anterior insula; anterior cingulate cortex, context-dependency 


\section{Introduction}

When a human being finds himself in a threatening situation together with another person, he notices his own fear but he also assesses the emotional state of mind of his conspecific. His brain processes self- versus other-related emotions at the same time. In a second step, he needs to weigh his own emotion to the emotion of others in order to decide if he flees or if he wants to help his threatened conspecific. In this situation, social animals include contextual factors in their decisions. The psychological construct of empathy, the capacity to understand or emotionally share what the other person experiences, enables us to react to the situation mentioned above: if we would not share the emotional state, namely fear, there would be no dilemma and the only one obvious reaction would be to help the person in need. Recent neuroscientific research has focused on brain regions involved in empathy and reported findings in the inferior parietal cortex, the anterior cingulate cortex (ACC), the premotor cortex and the anterior insula (AI)(Fan, Duncan, Greck, \& Northoff, 2011; Kral et al., 2018). These regions show increased activity when a person shares the emotion of someone else. The activity in these regions can be modulated by the neuropeptide oxytocin (OXT). OXT is both a hormone and a neurotransmitter, its effects can be measured in several areas of the brain, including the amygdala, the ACC and the Al. After the discovery that OXT induced maternal behavior in mice (Pedersen, Ascher, Monroe, \& Prange, 1982) and mate-attachment in prairie voles (Carter, Grippo, Pournajafi-Nazarloo, Ruscio, \& Porges, 2008), the focus of research shifted from the role of OXT in female reproduction towards its role in prosocial behavior in human beings. Intranasal OXT administration was found to increase trust towards strangers (Kosfeld, Heinrichs, Zak, Fischbacher, \& Fehr, 2005), decrease social anxiety and stress (Carter et al., 2008) and generally 
modulates social interaction behavior and social cognition in humans (Baumgartner, Heinrichs, Vonlanthen, Fischbacher, \& Fehr, 2008).

To further study the role of OXT in self- versus other-neuronal fear processing, we here examined the effect of OXT on regions related to empathy, namely cortical midline structures, the insular cortex and the amygdala. We hypothesized that OXT augments emotion sharing and therefore increases neuronal activity in these regions when a threatening stimulus is directed at another person, compared to when the same stimulus is directed at the subject itself. An augmentation of empathic feelings would then be related to a modulation of neuronal activity.

\section{Methods}

Participants and procedures

We investigated 28 healthy males aged 20 to 45 years. Participants in the oxytocin $(n=14,33.5 \pm 9.8$ years $)$ and placebo $(n=14,31.0 \pm 7.0$ years $)$ groups were agematched $(\mathrm{t}(27)=0.611, \mathrm{p}=0.547)$. All participants were right-handed as assessed by the Edinburgh Inventory for Handedness (Oldfield, 1971). The ability to understand study procedures was verified using the Multiple Choice Vocabulary Test (Lehrl, Triebig, \& Fischer, 1995), with an IQ $<90$ leading to exclusion. Participants with neurological, medical or psychiatric diseases, substance abuse and/or medication with potential central nervous system side effects (e.g., ß-blockers) were not included in the study. All procedures performed in the study were approved by the Ethics Committee Zurich (Project-Number E-53/2007) and are in accordance with the 1964 Helsinki declaration and its later amendments. All participants gave written informed consent to participate. One participant had to be excluded from the study due to extensive movement during the scanning procedure. 
Depression and anxiety scores were evaluated with the Beck Depression Scale (BDI), (Beck, Ward, Mendelson, Mock, \& Erbaugh, 1961), the State- Trait- AnxietyInventory (STAI),(Spielberger \& Vagg, 1984) and the General Well-being Questionnaire Bf-S (Zerssen, 1976). Empathy scores were assessed using the Interpersonal Reactivity Index (IRI) (Davis, 1983) and the Empathy- Quotient (EQ), (Baron-Cohen \& Wheelwright, 2004). These scales can be easily administered as a paper-and-pencil test and have previously been used in functional neuroimaging studies to predict empathy-related brain activation (Hassenstab, Dziobek, Rogers, Wolf, \& Convit, 2007; Schulte-Rüther, Markowitsch, Fink, \& Piefke, 2007; Singer et al., 2004).

Participants had to abstain from food and drink (other than water) for 2 hours before the experiment, and from exercise, caffeine, alcohol and nicotine during the 24 hours before the session. In a double-blind, between subjects, placebo-controlled study design they received a dose of 24 international units OXT (Syntocinon Spray, Novartis, Basel, Switzerland) or placebo (PLA) respectively, intranasally. All participants received either OXT $(n=14)$ or PLA $(n=14)$ approximately 45 min before the fMRI experiment.

\section{Experimental design}

Picture stimuli

We used 132 different emotional pictures with negative valence (norm ratings $2.24 \pm$ 2.67) from the International Affective Picture System (IAPS), (Lang, P.J., Bradley, M.M., \& Cuthbert, 2008). Pictures were balanced across conditions in terms of dominance, intensity, the total number of people, human faces and human figures as well as with respect to threats of natural origin (i.e. dogs, sharks) and threats of 
artificial origin (i.e. guns, plane crashes). The selection of pictures was based upon ratings by a separate group of 26 ( 11 male) participants who rated 164 pictures of the IAPS. They were instructed to rate subjective fear and empathy intensity on a nine-point visual analogue scale with 1 indicating very low intensity and 9 indicating high intensity. Participants could look at each picture on a computer screen until they had fully captured and internalized the content. They then responded on two visual analogue scales that appeared in random order. An ANOVA revealed differences of the fear intensity ratings between the pictures assigned to the fear condition $(n=87$, Median $=4, M=3.96 \pm 2.6)$ and the pictures assigned to the empathy condition $(n=$ 76, Median $=3, M=3.8 \pm 2.5 ; F(1,4252)=4.34, p=.037, \eta 2=.001)$. Calculations showed also strong differences of the empathy intensity ratings between the pictures assigned to the fear condition $(n=87$, Median $=3, M=3.54 \pm 2.6)$ and the pictures assigned to the empathy condition $(n=76$, Median $=6, M=5.42 \pm 2.7 ; F(1,4252)=$ $524.09, p=.000, \eta 2=.110)$. The fear condition in our experiment included only pictures showing threats directed at the observing participant. In the empathy condition, only pictures showing people threatened by human aggressors or natural dangers were included. Seventy-two neutral IAPS pictures (norm ratings $5.28 \pm 1.45$ ) were used as control, with 36 showing human faces or human figures, while the other 36 showed objects or animals. Control pictures were also balanced in terms of dominance and intensity. The experiment therefore constituted a $2 \times 3$ factorial design with the first factor representing 'substance' (OXT, PLA) and the second factor 'condition' (fear, empathy, control).

FMRI experiment 
The fMRI experiment consisted of 9 interleaved blocks of each of the 3 conditions.

Blocks consisted of 6 - 9 pictures and lasted between $30 \mathrm{sec}$ and $45 \mathrm{sec}$. The variable duration of blocks served to sample the BOLD response on as many time points as possible and to increase the sensitivity of the blocked design. All pictures were presented sequentially in a randomized fashion, with no inter-stimulus interval, for a period between $4.6 \mathrm{sec}$ and $5.8 \mathrm{sec}$. Nine blocks presenting a white fix-cross on grey background were inserted in random order. This design resulted in a total scan time of 23.8 minutes.

To assess the extent of fear and empathy, under each picture the two alternating questions "Fear?" and "Empathy?" randomly appeared. Participants were instructed to answer yes to the question "Fear?" and "Empathy?", respectively, if the threat depicted in the picture was either directed towards them or towards a third person. For the yes and no answers, participants used the left and right buttons of a 4-button response box. Participants had to give their response within the duration of the picture presentation. If no response was entered, an omission error was recorded in our presentation software (Neurobehavioral Systems, Inc.). Pictures were presented using MR-compatible video goggles (Resonance Technology Inc.).

\section{Behavioral monitoring}

Rating of the pictures was conducted outside the scanner immediately after the fMRI session. Each picture was presented for $2.8 \mathrm{~s}$ and fear and empathy intensity had to be rated on a nine-point scale. It is possible that emotional responses may attenuate when pictures are seen for a second time (Ishai, Pessoa, Bikle, \& Ungerleider, 2004). However, this habituation effect applies equally to both picture conditions and 
should not affect the differences between conditions (Anderson, C, Berkowitz, L, Donnerstein, E, Huesmann, L, Johnson, J, Linz, D, Malamuth, N, \& Wartella, 2003).

FMRI

Measurements were performed on a Philips Intera 3T whole-body MR unit equipped with an eight-channel Philips SENSE head coil. Functional time series were acquired with a sensitivity encoded single-shot echo-planar sequence (SENSE-sshEPI) (Pruessmann, Weiger, Scheidegger, \& Boesiger, 1999). The following acquisition parameters were used in the $\mathrm{fMRI}$ protocol: echo time $(\mathrm{TE})=35 \mathrm{msec}$, field of view $(\mathrm{FOV})=22 \mathrm{~cm}$, acquisition matrix $=80 \times 80$, interpolated to $128 \times 128$, voxel size 2.75 $\times 2.75 \times 4 \mathrm{~mm}^{3}$, SENSE acceleration factor $\mathrm{R}=2.0$. Using a midsagittal scout image, 32 contiguous axial slices were placed along the anterior-posterior commissure (ACPC) plane covering the entire brain with a repetition time $(T R)=3000 \mathrm{msec}(\mathrm{h}=$ $\left.82^{\circ}\right)$. Before the collection of the $\mathrm{fMRI}$ data we acquired a reference EPI scan for visual inspection of artefacts (e.g., ghosting). A 3D T1-weighted anatomical scan was obtained for structural reference.

Statistical analysis

Behavioral data

The scores of the questionnaires were compared between groups using t- tests for independent samples. The scores of the questionnaires within groups before and after the experiment were compared using t- tests for paired samples. The distribution of the yes and no answers of the fear and empathy assessment was tested with a chi-squared test for one sample over all conditions and with binomial tests for one sample between conditions. Reaction times of yes answers were 
analyzed using ANOVA and t- tests for independent samples. Post-scanning ratings of fear and empathy intensity were analyzed in a condition $x$ substance ANOVA. To investigate associations of BOLD data and behavioral data, we employed Pearson's product-moment correlation. We used a level of significance of .05. Statistical calculations were made using SPSS Statistic Software Version 25.

\section{FMRI Data \\ Preprocessing}

FMRI data was analyzed using Matlab 2015a (The Mathworks, Inc. 2015) and Statistical Parametric Mapping (SPM12, Welcome Trust Centre for Neuroimaging UCL, 2014). The images were corrected for differences in slice acquisition time, realigned to the first volume and normalized into standard stereotactic space. Images were smoothed using a Gaussian kernel of $6 \mathrm{~mm}$ full width at half maximum. Then, the data was linearly detrended and filtered by a band pass filter $(0.01-0.08 \mathrm{~Hz})$ to suppress cardiac and respiratory motion induced effects. Finally, time series data was controlled for global mean and motion outliers using Artifact Detection Tools (ART, The Gabrieli Lab. McGovern Institute for Brain Research, MIT, 2015).

\section{Whole brain analysis}

For the first level, condition effects were modeled by convolving a box-car epoch model with a canonical hemodynamic response function. For each subject, statistical parametric maps of the $t$ - statistics resulting from linear contrasts between the fear, empathy and control condition were calculated. The outliers of the motion statistics were entered in the model as covariates using the ART-software, but no motion 
regressors were used in our model. Differential contrasts of interest were fear vs. empathy, fear vs. control and empathy vs. control. These contrasts were calculated according to the experimental factors to assess differential modulation of the BOLD signal induced by each factor. For the between group analysis a full factorial design was used to model data of each participant with 2 factors which included substance (OXT, PLA) and condition (fear vs. empathy, fear vs. control and empathy vs. control). We report results that survive a statistical height threshold of $p<.05$, familywise error (FWE) corrected for multiple comparisons on cluster level, and a minimum cluster extent of at least 40 voxels.

\section{$\mathrm{ROI}$ analysis}

For those brain regions targeted by a priori hypotheses, a region-of interest (ROI) approach was applied. The ROls were chosen based on meta-analyses on Neurosynth. This is a platform for large scale, automated synthesis of fMRI data (http://neurosynth.org). We searched on the terms "empathy" (137 studies) and "fear" (298 studies). The regions selected are: anterior and posterior cingulate $(x=0, y=$ 22, $z=-10 ; x=0, y=-48, z=28)$, bilateral amygdala $(x=22, y=-26, z=-2 ; x=26$, $y=-2, z=-22)$, bilateral Al $(x=-40, y=20, z=2 ; x=40, y=20, z=2)$, bilateral posterior insula (PI; $x=-40, y=-2, z=12 ; x=40, y=2, z=12$ ) and bilateral temporo-parietal junction $(x=-46, y=-50, z=20 ; x=46, y=-50, z=20)$. We defined ROls as $10 \mathrm{~mm}$ spheres from the above-mentioned coordinates, an exception being the coordinates of the amygdala, where the radius of the sphere was reduced to $5 \mathrm{~mm}$. For each $\mathrm{ROI}$ we extracted the beta values for each participant for the contrasts of interest using the MarsBaR Toolbox for SPM. For within group comparisons between Fear, Empathy and Control conditions, we 
calculated t- tests for paired samples for each ROI. Between groups analyses for each ROI were calculated using ANOVA.

\section{Results}

Behavioral data

The IQ scores measured with the MWT did not differ between the OXT (M = 117.8, $S D=19.1)$ and PLA group $\left(M=109.4, S D=9.8 ; F(1,25)=1.90, p=.181, \eta^{2}=\right.$ .076). All other scores of the questionnaires are summarized in table 1 . There were no significant differences between the OXT and the PLA group. The state anxiety scores before and after the experiment also did not differ. The extent of fear and empathy was assessed during the experiment. During the fear and the empathy condition, more "yes" answers were given to the fear assessment than in the control condition. The omission rate was low across all conditions in the PLA and the OXT group (see table 2). In the empathy condition, more "yes" answers were given to the empathy assessment than in the fear and in the control condition. This pattern of differences was shown in the PLA and the OXT group (Table 2). Reaction times of the yes answers were analyzed using a 2 (Group: OXT vs. PLA) x 2 (Condition: Fear vs. Empathy) ANOVA. There were main effects of medication $(F(1,1184)=4.76, p=$ $\left..029, n p^{2}=.004\right)$ and condition $\left(F(1,1184)=25.38, p=.000, n p^{2}=.021\right)$ as well as a significant interaction $\left(F(1,1184)=4.87, p=.027, n p^{2}=.004\right)$. Posthoc tests showed increased reaction times for the "yes" answers in the OXT group compared to the PLA group in the empathy condition $\left(F(1,510)=10.11, p=.002, \eta^{2}=.020\right)$, but not in the fear condition (supplemental table 1).

A One-Way ANOVA of the fear intensity ratings immediately after the fMRI experiment showed no substance effect $(F(1,3743)=.11, p=.737, \eta 2=.000)$, but a 
significant effect of the conditions $(F(2,3741)=460.13, p=.000, \eta 2=.198)$. Posthoc calculations revealed differences $(p \leq .001)$ of the conditions fear $(O X T, M=4.14 \pm$ 2.4; PLA, $M=4.06 \pm 2.7)$, empathy (OXT, $M=3.36 \pm 2.3 ; P L A, M=3.60 \pm 2.6)$ and control (OXT, $M=1.48 \pm 1.1 ; \mathrm{PLA}, \mathrm{M}=1.40 \pm 1.1)$. The calculation of the empathy intensity ratings after the experiment showed a substance effect $(F(1,3743)=$ $57.730, p=.000, \eta 2=.015)$ and an effect of the conditions $(F(2,3741)=495.54, p=$ $.000, \eta 2=.210)$. Posthoc Bonferroni tests revealed differences $(p \leq .001)$ of the conditions fear (OXT, $M=2.62 \pm 2.2 ; \mathrm{PLA}, \mathrm{M}=3.20 \pm 2.6$ ), empathy (OXT, $M=5.06$ $\pm 2.6 ; \mathrm{PLA}, \mathrm{M}=5.85 \pm 2.6)$ and control $(\mathrm{OXT}, \mathrm{M}=2.17 \pm 1.8 ; \mathrm{PLA}, \mathrm{M}=2.71 \pm 2.4$ ).

\section{FMRI Data}

Whole brain analysis

Significant activation patterns $(p<.05$, FWE corrected) are listed in table 3 and shown in figure 2.

\section{$\mathrm{ROI}$ analysis}

Within group comparisons

Significant differences between conditions were found only in the OXT, but not in the PLA group. Paired sampled t-tests revealed higher activation in the fear condition compared to the control condition in bilateral temporo-parietal junction (left, $t(13)=$ 2.232, $\mathrm{p}=.044$, dcohen $=.4$; right, $\mathrm{t}(13)=2.252, \mathrm{p}=.042$, dcohen $=.6)$. Higher activation in the fear condition could also be found in the right $\mathrm{Al}(\mathrm{t}(13)=2.330, \mathrm{p}=$ .037 , dcohen $=.7)$ and left posterior insula $(\mathrm{t}(13)=3.127, \mathrm{p}=.008$, dcohen $=.8)$.

Between group comparisons 
Comparisons using 2 (Group: OXT vs. PLA) x 3 (Condition: Fear, Empathy, Control) ANOVAs revealed differences between the OXT and PLA groups in the ACC (F (2, $\left.25)=3.74, p=.031, n p^{2}=.130\right)$ and the right $\operatorname{Al}\left(F(2,25)=4.22, p=.020, n p^{2}=\right.$ .145). Post -hoc t-tests showed in the ACC a between group effect in the fear condition $\left(\mathrm{t}(25)=2.430, \mathrm{p}=.023, \mathrm{~d}_{\text {cohen }}=.9\right)$ with stronger activation in the OXT group. Differences in the empathy condition between OXT and PLA in the right AI reached only marginal significance $(\mathrm{t}(25)=1.940, \mathrm{p}=.064$, dcohen $=.7)$ with stronger activation in the PLA group.

Correlations between behavioral and BOLD data Empathy scores

In the empathy condition, activation in the right PI correlated negatively with the EQ / IRI scores in the PLA group $(r=-.59, p=.035 / r=-.69, p=.009)$, but not in the OXT group. This indicates higher brain activity in participants with lower empathy scores. In the OXT group a positive correlation between the EQ / IRI scores and the activation in the left amygdala $(r=.62, p=.023 / r=.60, p=.032)$ was found in the empathy condition indicating higher brain activity in participants with higher empathy scores. No correlations between BOLD data and empathy scores were found in the fear condition. See table 4 for further details.

\section{Anxiety scores}

In the fear condition, negative correlations between trait anxiety and bilateral Al activation (left, $r=-.57, p=.041$; right, $r=-.83, p=.001$ ) could be found in the OXT group. This indicates higher insular activity with OXT in generally less anxious participants. No correlations were found between trait anxiety scores and BOLD data 
in any condition. See table 4 and figure 2 for details.

\section{Discussion}

The aim of this study was to investigate whether OXT administration influences the processing of self- compared to other-related fear in a way that it augments the activity in brain regions that are important for other-related fear processing. Our results show that OXT increases activity in the ACC in the fear compared to the control condition. Another finding in our data is that when a threat is directed at another individual, activity of the Al tends to decrease in the OXT group compared to the PLA group. These findings are contradictory to our hypothesis: We assumed that OXT would increase activity in the Al and the ACC, when a threat is directed at someone else compared to when a threat is directed at oneself, which would have confirmed the role of OXT in intensifying the processing of other-related fear.

The effect of OXT as a prosocial hormone has been questioned by several studies and meta-analyses. In animals, Rogers-Carter and colleagues found that blockade of OXT receptors in the Al leads to a change in age-dependent approach vs. avoidance behavior of rats towards a conspecific (Rogers-Carter et al., 2018). Usually, being confronted with a scared conspecific, the rat would approach a young individual, while reacting aggressively to an older rat. This preference to younger conspecific can be inhibited by blocking the OXT receptors in the Al, thus leading to a lower activity in the $\mathrm{Al}$ and thereby inducing an approach to the older rat. Additionally, male rats approached familiar conspecifics more likely while reacting aggressively towards unfamiliar individuals. This behavior could not be detected in female rats. In humans it could be shown that OXT increases prosocial behavior towards safe stimuli (when a familiar conspecific is present) and defensiveness towards unsafe stimuli 
(Leppanen, Ng, Tchanturia, \& Treasure, 2017). A systematic review conducted by

Olff and colleagues states that OXT increases sensitivity to social salience cues, but that the final effect is moderated both by contextual factors (e.g., if a familiar person is present) and by interindividual factors (e.g., sex, presence of a psychiatric disease etc.) (Olff et al., 2013). In threatening and competitive situations, OXT may therefore have an aggressive and competitive effect rather than a prosocial effect (Cohen and Shamay-Tsoory, 2017). Furthermore, there are studies that state that OXT receptor methylation and polymorphisms of the OXT receptor gene influence the effect of OXT. Higher OXT receptor methylation leads to higher activity in the regions which express OXT receptors, also in regions known to be active in social behavior, such as ACC and the Al (Puglia et al., 2015). On the other hand, it was found that a variant of the OXT receptor gene (rs237915) leads to lower responsiveness to negative social emotional cues, due to diminished activity in the ventral striatum (Loth et al., 2014). This suggests that both epigenetic as well as genetic factors affect the impact of OXT on certain brain regions.

The here reported results in male participants cannot be generalized to female subjects. Females perform better in emotion recognition, show advantages in theory of mind and are more empathic and thus more altruistic than males (Christov-Moore et al., 2016). Accordingly, if we had conducted this study with female instead of male participants, a greater difference between conditions might have emerged. Luo and colleagues found that in males, OXT suppressed activation in the inferior frontal gyrus, the dorsal $A C C$ and the $A I$ in response to threatening face stimuli, but increased activation in these regions in females. Additionally, Lynn et al. found that OXT impairs males' ability to optimally adapt their emotion perception skills to differences in risk and uncertainty that characterize different social contexts (Lynn, 
Hoge, Fischer, Feldman, \& Simon, 2014). Female participants however, showed no difference in their adaption capacity towards angry faces whether they received OXT or PLA. On the other hand, Domes et al. found that OXT increases the activity in the amygdala in females, when a human face expresses fear. The same effect could not be detected in males (Domes et al., 2010). Due to limited sample size in contemporary fMRI studies, the inclusion of sex as a factor in the statistical analysis is challenging, but essential in future research about the influence of OXT on the social brain. In summary, results of recent studies, including our own, lead to the conclusion that OXT does not always lead to a higher valence of other-related fear compared to self-related fear, but that its effect is depending on the context factors. Regarding the effect of OXT on the fear circuit, Eckstein and colleagues reported that OXT has an anxiolytic effect due to dampened amygdala activity (Eckstein et al., 2016). Consistently, higher levels of OXT correlate with less stress and anxiety, which are in turn related to decreased activation of the amygdala and the medial prefrontal cortex (Lahoud \& Maroun, 2013). The influence of OXT leads to a significantly reduced activity in both the ACC and the AI in context fear conditioning compared to cue fear conditioning (Cavalli et al., 2018). This suggests that the effect of OXT on the ACC and the Al is important for the processing of fearful signals in a social context.

The processing of fear has long been a research focus. Recently, separate circuits of fear and anxiety processing were proposed (LeDoux, 2015). Following this concept, Smith and Lane constructed a framework about emotion trigger factors and recognition and differed between cognitive emotions and embodied emotions (Smith \& Lane, 2015). In the first case, thoughts and personal experience trigger an emotion, whereas in the second case, bodily feelings lead to an emotion. Regardless 
of what triggers the emotion, both cases lead to a change in somatic features, for example an increase in heart rate. These somatic features are registered by afferent nuclei in the brainstem and then need to be interpreted by the AI. It generates a whole-body pattern, in which specific bodily feelings correspond to a specific emotion. The ACC on the other hand is important for the conscious experience of the feeling and the cognitive recognition of the emotion. In our study, the activation in the Al tended to decrease when the threat was directed at another person, probably due to missing bodily sensations. The activation of the ACC however stayed the same because the cognitive recognition of the emotion still took place.

In conclusion, this study supports current research questioning that OXT has exclusively prosocial effects on human behavior. The results in this study show how difficult it is to assess the connections between anxiety processing, social behavior, and humoral influences. Further research should concentrate on contextual and individual factors, which may influence the effect of OXT and on the effect of OXT in females.

\section{Limitations}

In this section, a number of methodological issues related to the acquisition of the data and the significance of the used measures are discussed. First, we decided to investigate the effect of OXT only in male participants. The inclusion of female participants would have required the extensive control of confounding factors such as menstrual cycle or hormonal contraception. Furthermore, as stated above, social abilities, the influence of OXT and neuronal processing of social situations differ considerably between male and females (Domes et al., 2010; Lynn et al., 2014). To elucidate the sex-dependent influences of OXT on the neuronal processing of social 
situations, future investigations should however study male and female participants.

Second, our study might be underpowered. In the future, our results should be replicated in a larger sample. Third, we only applied mild stimuli using standardized fear pictures. The study could be replicated with pictures matching each subject's fears or with fearful movies, to generate a more realistic frightening environment. Furthermore, stimuli with an individualized interpersonal context could be used to intensify the relational aspects of an experiment and a baseline OXT serum level of each participant should be measured. Fourth, even though the pictures in the experiments were balanced across conditions in terms of their contents and separately rated before the experiment, they were not controlled for context dependency of stimuli. We did not control if the pictures showed only threatening features, or might as well have included some soothing features. Subsequent studies should control for context dependency. Fifth, attentiveness to stimuli towards oneself might have been higher compared to attentiveness when the stimuli were directed at someone else. This might have led to higher activity in the ROls when a threat was directed at oneself. However, at least in the PLA group, reaction times did not differ between the fear and empathy condition. Finally, one has to consider that by naming their emotional state of mind, the participants conducted a cognitive task, which might have been a distractor.

\section{Conflict of interest}

Linda Bättig declares that she has no conflict of interest. Andreas Baeumelt declares that he has no conflict of interest. Jutta Ernst declares that she has no conflict of interest. Heinz Boeker declares that he has no conflict of interest. Simone Grimm declares that she has no conflict of interest. Andre Richter declares 
that he has no conflict of interest.

\section{Tables and Figures}

Table 1: Questionnaire data as mean scores \pm standard deviation (range) for the OXT and PLA groups.

Table 2: Responses to the assessment of the presence of fear or empathy during the picture presentation. Note that fear but not empathy was reported in both conditions compared to the control condition in the OXT and PLA groups.

Table 3: Cluster of activation reaching criteria of significance in the whole brain analysis in the OXT group and in the comparison of the groups.

Table 4: Correlations between BOLD data and the scores of the questionnaires in participants with placebo and oxytocin

Figure 1: Schematic representation of the experimental design. The experiment had the conditions fear, empathy and control; here indicated with the colors red, green and blue (A). Each trial consisted of an IAPS-picture combined with the question "Fear?" or "Empathy?" and lasted between 4.6 seconds and 5.8 seconds (no interstimulus interval). Six to 9 trials of the same condition were presented in blocks in a randomized order with durations between 30 seconds and 45 seconds. In summary, a number of 27 blocks were presented (B). Between the conditions, nine blocks with a duration between 30 and 45 seconds presenting a white fix-cross on grey background were inserted in random order. The experiment lasted a total of 
23.8 minutes. Please note that due to copyright regulations, the images may only be displayed in a blurred form.

Figure 2: Voxel-wise, wholebrain analysis $(\mathrm{A})$ with higher $\mathrm{ACC}$ activation in the Fear compared to the Empathy and the Control condition, thresholded using clustersize > 40 voxel and FWE corrected P-value <.05. The underlay and coordinates are in MNI space. ROI analysis of ACC (B) and bilateral insula (C and D) showing the mean percent signal change in the OXT and PLA group for all conditions. Error bars represent $95 \%$ Cls above and below the mean. Significant differences between conditions are indicated by the exact $p$-value. Significant differences and trends in the post -hoc t-tests of the between group comparisons are also indicated by the exact $p$-value. The scatter plots show the relationship between individual trait anxiety scores (STAI-X2) obtained from each subject in the OXT group and the signal change in the fear condition in the right $(C)$ and left $(D)$ anterior insula.

\section{References}

Anderson, C, Berkowitz, L, Donnerstein, E, Huesmann, L, Johnson, J, Linz, D, Malamuth, N, \& Wartella, E. (2003). The influence of media violence on youth: Psychological Science In The Public Interest, 4(3), 81-110. https://doi.org/10.1111/j.1529-1006.2003.pspi_1433.x

Baron-Cohen, S., \& Wheelwright, S. (2004). The empathy quotient: An investigation of adults with asperger syndrome or high functioning autism, and normal sex differences. Journal of Autism and Developmental Disorders, 34(2), 163-175. https://doi.org/10.1023/B:JADD.0000022607.19833.00

Baumgartner, T., Heinrichs, M., Vonlanthen, A., Fischbacher, U., \& Fehr, E. 
(2008).Oxytocin Shapes the Neural Circuitry of Trust and Trust Adaptation in Humans, 639-650. https://doi.org/10.1016/j.neuron.2008.04.009

Beck, A. T., Ward, C. H., Mendelson, M., Mock, J., \& Erbaugh, J. (1961). An inventory for measuring depression. Archives of General Psychiatry, 4, 561571.

Carter, C. S., Grippo, A. J., Pournajafi-Nazarloo, H., Ruscio, M. G., \& Porges, S. W. (2008). Oxytocin, vasopressin and sociality. Progress in Brain Research, 170(08), 331-336. https://doi.org/10.1016/S0079-6123(08)00427-5

Cavalli, J., Ruttorf, M., Pahi, M. R., Zidda, F., Flor, H., \& Nees, F. (2018). Oxytocin differentially modulates pavlovian cue and context fear acquisition. Social Cognitive and Affective Neuroscience, 976-983. https://doi.org/10.1093/scan/nsx028

Christov-Moore, L., Simpson, E. A., Grigaityte, K., lacoboni, M., Ferrari, P. F., (2016). Empathy: Gender effects in brain and behavior. Neuroscience and Biobehavioral Reviews (Vol. 46). https://doi.org/10.1016/j.neubiorev.2014.09.001.Empathy

Cohen, D., \& Shamay-Tsoory, S. G. (2017). Oxytocin regulates social approach. Social Neuroscience, 13(6), 680-687. https://doi.org/10.1080/17470919.2017.1418428

Davis, M. H. (1983). Measuring individual differences in empathy: evidence for a multidimensional approach. J. Pers. Soc. Psychol., 44, 113-126.

Domes, G., Lischke, A., Berger, C., Grossmann, A., Hauenstein, K., Heinrichs, M., \& Herpertz, S. C. (2010). Effects of intranasal oxytocin on emotional face processing in women. Psychoneuroendocrinology, 35(1), 83-93 https://doi.org/10.1016/j.psyneuen.2009.06.016 
Eckstein, M., Scheele, D., Patin, A., Preckel, K., Becker, B., Walter, A., ...

Hurlemann, R. (2016). Oxytocin Facilitates Pavlovian Fear Learning in Males.

Neuropsychopharmacology, 41(4), 932-939.

https://doi.org/10.1038/npp.2015.245

Fan, Y., Duncan, N. W., Greck, M. De, \& Northoff, G. (2011). Is there a core neural network in empathy? An fMRI based quantitative. Neuroscience and Biobehavioral Reviews, 35(3), 903-911.

https://doi.org/10.1016/j.neubiorev.2010.10.009

Hassenstab, J. M. A., Dziobek, I. P., Rogers, K. M. A., Wolf, O. T. P., \& Convit, A. M. D. (2007). Knowing What Others Know, Feeling What Others Feel: A Controlled Study of Empathy in Psychotherapists. Journal of Nervous \& Mental Disease, 195(4), 277-281.

Ishai, A., Pessoa, L., Bikle, P. C., \& Ungerleider, L. G. (2004). Repetition suppression of faces is modulated by emotion. Proceedings of the National Academy of Sciences of the United States of America, 101(26), 9827-9832. https://doi.org/10.1073/pnas.0403559101

Kosfeld, M., Heinrichs, M., Zak, P. J., Fischbacher, U., \& Fehr, E. (2005). Oxytocin increases trust in humans. Nature, 435, 673-676.

https://doi.org/10.1038/nature03701

Kral, T. R. A., Solis, E., Mumford, J. A., Schuyler, B. S., Flook, L., Rifken, K., ... Madison, W. (2018). Neural correlates of empathic accuracy in adolescence. Social Cognitive and Affective Neuroscience, 1701-1710. https://doi.org/10.1093/scan/nsx099

Lahoud, N., \& Maroun, M. (2013). Oxytocinergic manipulations in corticolimbic circuit differentially affect fear acquisition and extinction. Psychoneuroendocrinology, 
38(10), 2184-2195. https://doi.org/10.1016/j.psyneuen.2013.04.006

Lang, P.J., Bradley, M.M., \& Cuthbert, B. N. (2008). International affective picture system (IAPS): Affective ratings of pictures and instruction manual. Gainesville, FL.

LeDoux, J. (2015). Anxious: Using the Brain to Understand and Treat Fear and Anxiety. New York: Viking.

Lehrl, S., Triebig, G., \& Fischer, B. (1995). Multiple choice vocabulary test MWT as a valid and short test to estimate premorbid intelligence. Acta Neurologica Scandinavica, 91(5), 335-345. https://doi.org/10.1111/j.16000404.1995.tb07018.x

Leppanen, J., Ng, K. W., Tchanturia, K., \& Treasure, J. (2017). Meta-analysis of the effects of intranasal oxytocin on interpretation and expression of emotions. Neuroscience and Biobehavioral Reviews, 78, 125-144. https://doi.org/10.1016/j.neubiorev.2017.04.010

Luo, L., Becker, B., Geng, Y., Zhao, Z., Gao, S., Zhao, W., ... Kendrick, K. M. (2017). Sex-dependent neural effect of oxytocin during subliminal processing of negative emotion faces. Neurolmage, 162, 127-137. https://doi.org/10.1016/j.neuroimage.2017.08.079

Lynn, S. K., Hoge, E. A., Fischer, L. E., Feldman, L., \& Simon, N. M. (2014). Gender differences in oxytocin-associated disruption of decision bias during emotion perception. Psychiatry Research, 219(1), 198-203. https://doi.org/10.1016/j.psychres.2014.04.031

Oldfield, R. C. (1971). The assessment and analysis of handedness: The Edinburgh inventory. Neuropsychologia, 9(1), 97-113. https://doi.org/10.1016/00283932(71)90067-4 
Olff, M., Frijling, J. L., Kubzansky, L. D., Bradley, B., Ellenbogen, M. A., Cardoso, C., ... Zuiden, M. Van. (2013). The role of oxytocin in social bonding , stress regulation and mental health : An update on the moderating effects of context and interindividual differences. Psychoneuroendocrinology, 38(9), 1883-1894. https://doi.org/10.1016/j.psyneuen.2013.06.019

Pedersen, C. A., Ascher, J. A., Monroe, Y. L., \& Prange, A. J. (1982). Oxytocin induces maternal behavior in virgin female rats. Science, 216(4546), 648-650. https://doi.org/10.1126/science.7071605

Pruessmann, K. P., Weiger, M., Scheidegger, M. B., \& Boesiger, P. (1999). SENSE: sensitivity encoding for fast MRI. Magn Reson.Med., 42(5), 952-962.

Rogers-Carter, M. M., Varela, J. A., Gribbons, K. B., Pierce, A. F., McGoey, M. T., Ritchey, M., \& Christianson, J. P. (2018). Insular cortex mediates approach and avoidance responses to social affective stimuli. Nature Neuroscience, 21(3), 404-414. https://doi.org/10.1038/s41593-018-0071-y

Schulte-Rüther, M., Markowitsch, H. J., Fink, G. R., \& Piefke, M. (2007). Mirror neuron and theory of mind mechanisms involved in face-to-face interactions: $A$ functional magnetic resonance imaging approach to empathy. Journal of Cognitive Neuroscience, 19(8), 1354-1372. https://doi.org/10.1162/jocn.2007.19.8.1354

Singer, T., Seymour, B., O’Doherty, J., Kaube, H., Dolan, R. J., \& Frith, C. D. (2004). Empathy for Pain Involves the Affective but not Sensory Components of Pain. Science, 303(5661), 1157-1162.

Smith, R., \& Lane, R. D. (2015). Neuroscience and Biobehavioral Reviews The neural basis of one 's own conscious and unconscious emotional states. Neuroscience and Biobehavioral Reviews, 57, 1-29. 
https://doi.org/10.1016/j.neubiorev.2015.08.003

Spielberger, C. D., \& Vagg, P. R. (1984). Psychometric properties of the STAI: a reply to Ramanaiah, Franzen, and Schill. J Pers Assess, 48(1), 95-97.

Zerssen, D. (1976). Die Befindlichkeitsskala Bf-S. Weinheim: Beltz. 
A

Block Fear

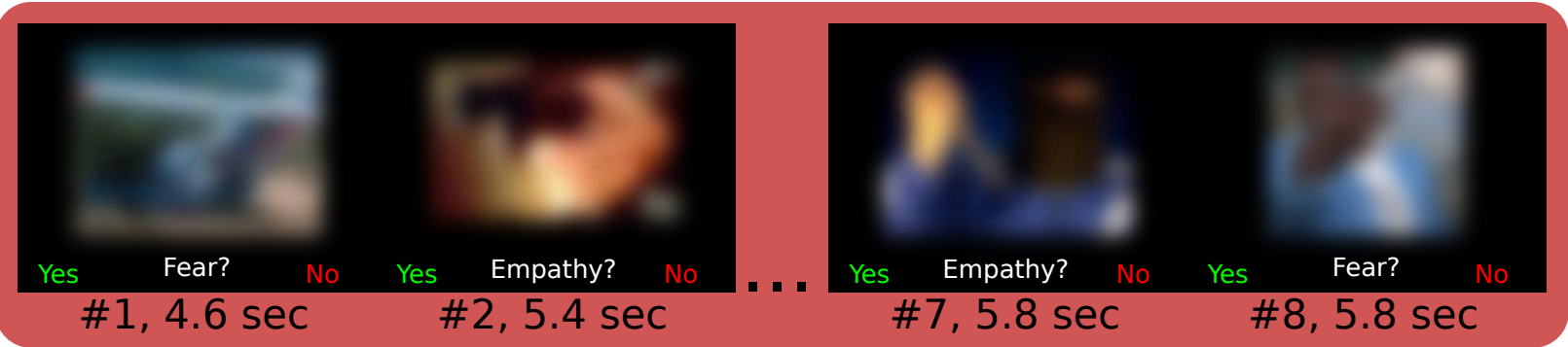

Block Empathy

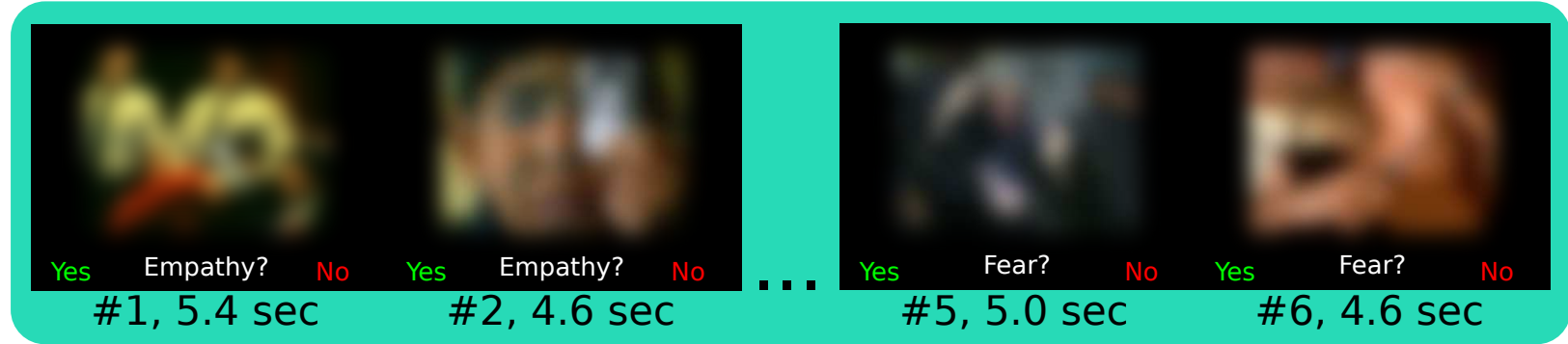

\section{Block Control}

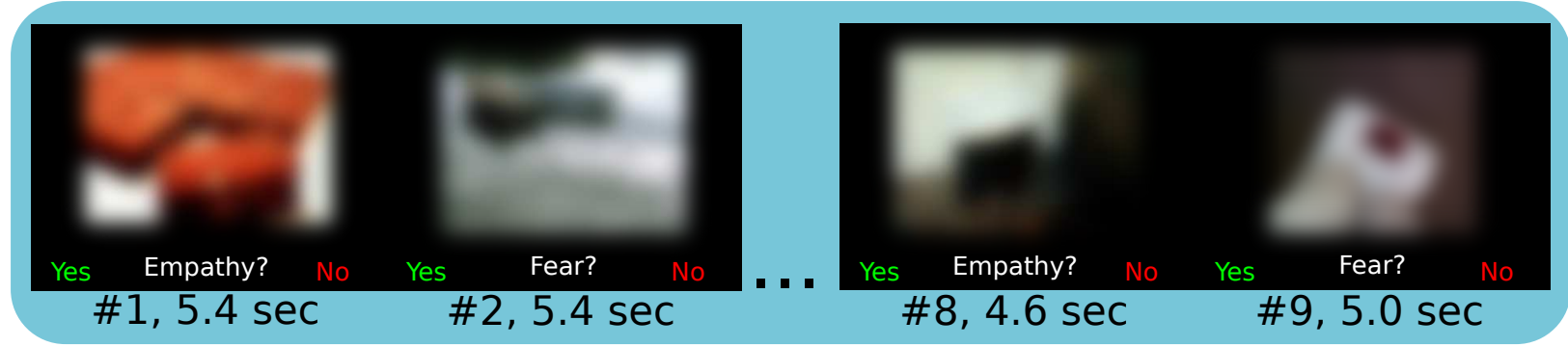

B 


\section{A OXT}

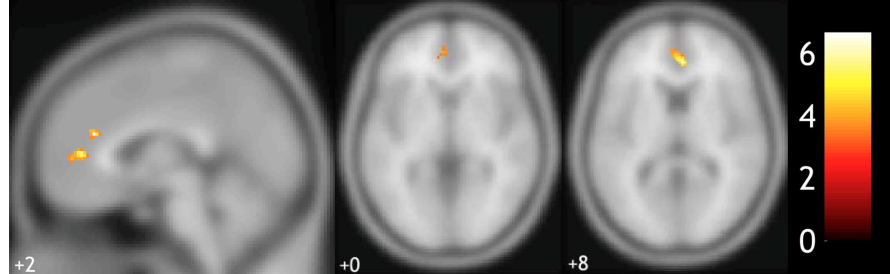

Fear > Empathy

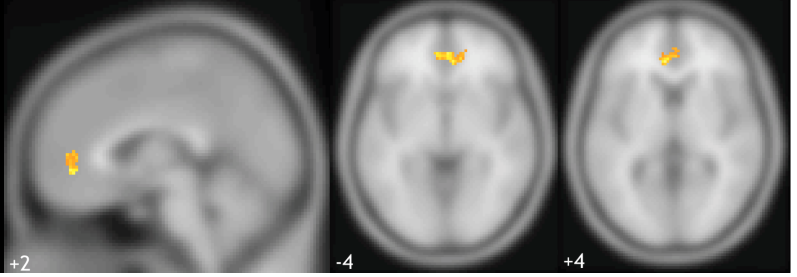

Fear > Control

B Anterior cingulate cortex

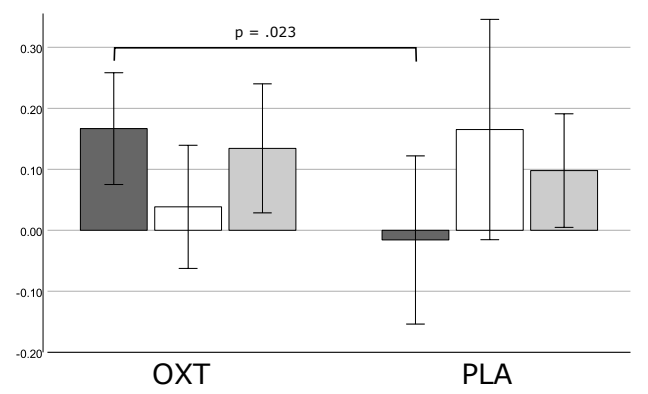

Legend

$\square$ Fear

$\square$ Empathy

Control

\section{Right anterior insula}
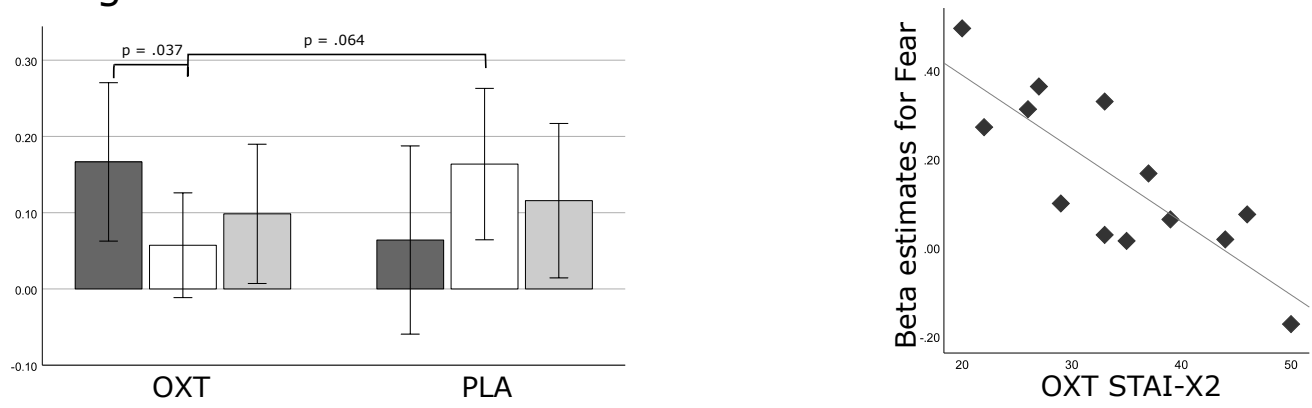

D Left anterior insula
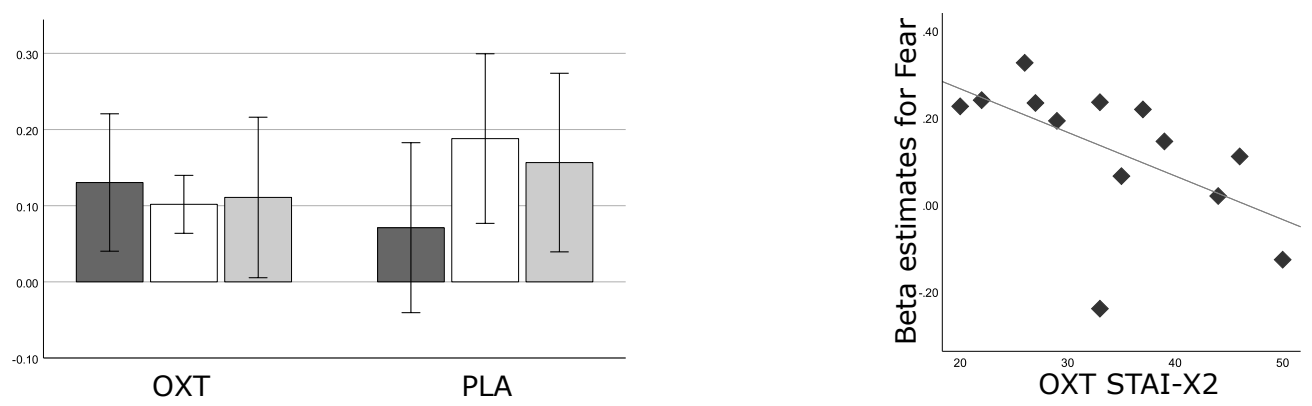


\begin{tabular}{llllllll}
\hline Questionnaire & \multicolumn{2}{l}{ OXT $(\mathbf{n = 1 4 )}$} & PLA $(\mathbf{n}=\mathbf{1 3})$ & \multicolumn{2}{l}{ T Test } & \\
& & & & $\mathrm{t}$ & Sig. & $\mathrm{Eta}^{2}$ \\
\hline BDI total & $3.5 \pm 2$ & $(0-6)$ & $5.1 \pm 7$ & $(0-22)$ & .777 & .445 & .025 \\
EQ & $32.9 \pm 11$ & $(15-55)$ & $36.8 \pm 12$ & $(21-54)$ & .871 & .392 & .031 \\
IRI & $49.8 \pm 8$ & $(21-54)$ & $45.2 \pm 6$ & $(38-55)$ & 1.918 & .069 & .133
\end{tabular}

STAl state

$\begin{array}{llllllll}\text { Pre } & 35.1 \pm 9 & (24-56) & 32.9 \pm 9 & (20-53) & -.655 & .519 & .018\end{array}$

$\begin{array}{llllllll}\text { Post } & 34.9 \pm 9 & (25-58) & 31.8 \pm 7 & (23-43) & -1.015 & .321 & .042\end{array}$

$\begin{array}{llllllll}\text { STAl trait } & 33.9 \pm 9 & (22-49) & 35.5 \pm 9 & (24-53) & .440 & .664 & .001\end{array}$

Bf-S

$\begin{array}{llllllll}\text { Pre } & 45.5 \pm 7 & (29-56) & 44.0 \pm 12 & (19-56) & -.372 & .713 & .006 \\ \text { Post } & 44.6 \pm 9 & (26-56) & 45.3 \pm 10 & (27-56) & .209 & .836 & .002\end{array}$

$\mathrm{BDI}=$ Beck Depression Inventory, EQ = Empathy Quotient, IRI = Interpersonal Reactivity Index, STAI = State-Trait Anxiety Inventory, Bf-S = General Well-being Questionnaire 


\begin{tabular}{|c|c|c|c|c|c|c|c|c|}
\hline \multicolumn{2}{|c|}{ Assessment } & \multicolumn{2}{|l|}{ Fear } & \multicolumn{2}{|c|}{ Empathy } & \multicolumn{2}{|c|}{ Control } & \multirow{2}{*}{$\begin{array}{l}\text { Chi- } \\
\text { Square } \\
\text { Test }\end{array}$} \\
\hline & & $\mathrm{n}$ & $\%$ & $n$ & $\%$ & $\mathrm{n}$ & $\%$ & \\
\hline \multicolumn{9}{|l|}{ PLA } \\
\hline \multirow[t]{3}{*}{ Fear? } & Yes & $200^{\#}$ & 50.5 & $245^{\S}$ & 55.2 & 8 & 2.1 & .000 \\
\hline & No & 191 & 48.2 & 193 & 43.4 & 375 & 97.6 & \\
\hline & Omissions & 5 & 1.3 & 6 & 1.4 & 1 & .3 & \\
\hline \multirow[t]{3}{*}{ Empathy? } & Yes & $163^{*}$ & 38.8 & $261^{\S}$ & 70.2 & 130 & 27.1 & .000 \\
\hline & No & 256 & 61.0 & 105 & 28.2 & 346 & 72.1 & \\
\hline & Omissions & 1 & .2 & 6 & 1.6 & 4 & .8 & \\
\hline \multicolumn{9}{|l|}{ OXT } \\
\hline \multirow[t]{3}{*}{ Fear? } & Yes & $225^{\#}$ & 52.5 & $238^{\S}$ & 49.5 & 43 & 10.4 & .000 \\
\hline & No & 197 & 45.9 & 228 & 47.4 & 365 & 87.7 & \\
\hline & Omissions & 7 & 1.6 & 15 & 3.1 & 8 & 1.9 & \\
\hline \multirow[t]{3}{*}{ Empathy? } & Yes & $151^{*}$ & 33.2 & $249 \S$ & 61.8 & 149 & 28.7 & .000 \\
\hline & No & 299 & 65.7 & 145 & 36.0 & 359 & 69.0 & \\
\hline & Omissions & 5 & 1.1 & 9 & 2.2 & 12 & 2.3 & \\
\hline
\end{tabular}

* One-Sample Binomial Test Fear vs. Empathy $p \leq .001$

\# One-Sample Binomial Test Fear vs. Control $p \leq .001$

$\S$ One-Sample Binomial Test Empathy vs. Control $p \leq .001$ 


\begin{tabular}{|c|c|c|c|c|c|c|c|c|c|}
\hline \multirow[t]{2}{*}{ Contrast } & \multirow[t]{2}{*}{ Region } & \multirow[t]{2}{*}{ BA } & \multirow[t]{2}{*}{ Cluster size } & \multirow{2}{*}{$\begin{array}{c}\text { FWE } \\
\text { corrected P- } \\
\text { value } \\
\text { (cluster-level) }\end{array}$} & \multirow[t]{2}{*}{ T-value } & \multirow{2}{*}{$\begin{array}{c}\text { FWE } \\
\text { corrected P. } \\
\text { value } \\
\text { (voxel-level) }\end{array}$} & \multicolumn{3}{|c|}{$\begin{array}{c}\text { MNI- } \\
\text { Coordinates } \\
\text { (peaks) }\end{array}$} \\
\hline & & & & & & & $\mathbf{x}$ & $y$ & $\mathbf{z}$ \\
\hline \multicolumn{10}{|l|}{ OXT } \\
\hline Fear > Control & ACC & $10 / 32$ & 198 & 0.000 & 6.66 & 0.684 & 12 & 42 & -2 \\
\hline Fear > Empathy & ACC & 32 & 112 & 0.005 & 6.62 & 0.651 & 4 & 40 & 8 \\
\hline \multicolumn{10}{|l|}{ PLA > OXT } \\
\hline \multirow[t]{2}{*}{ Empathy > Control } & Right putamen & & 130 & 0.012 & 4.98 & 0.637 & 16 & 10 & -10 \\
\hline & Right PMC & 6 & 116 & 0.021 & 4.76 & 0.948 & 52 & -2 & 28 \\
\hline
\end{tabular}

$\mathrm{ACC}=$ Anterior cingulate cortex, $\mathrm{PMC}=$ Primary motor cortex, FWE = Familywise error correction, $\mathrm{MNI}=\mathrm{Montreal}$

Neurological Institute, BA = Brodmann Area 


\begin{tabular}{|c|c|c|c|c|c|c|c|}
\hline \multirow[t]{2}{*}{ Medication } & \multirow[t]{2}{*}{ Brain region } & \multirow{2}{*}{$\begin{array}{r}\text { EQ } \\
r\end{array}$} & \multicolumn{3}{|c|}{ IRI } & \multicolumn{2}{|c|}{ STAI trait } \\
\hline & & & Sig. & $r$ & Sig. & $r$ & Sig. \\
\hline \multicolumn{8}{|l|}{ Placebo } \\
\hline & Empathy & & & & & & \\
\hline & Right posterior insula & -.587 & .035 & -.693 & .009 & & \\
\hline \multicolumn{8}{|l|}{ Oxytocin } \\
\hline & Empathy & & & & & & \\
\hline & Left amygdala & .623 & .023 & .596 & .032 & & \\
\hline & Anterior cingulate & & & -.647 & .017 & & \\
\hline & Fear & & & & & & \\
\hline & Left anterior insula & & & & & -.571 & .041 \\
\hline & Right anterior insula & & & & & -.825 & .001 \\
\hline & Neutral & & & & & & \\
\hline & Anterior cingulate & & & -.626 & .022 & & \\
\hline
\end{tabular}

